\title{
Improved dietary intake among overweight and obese children followed from 8 to 12 years of age in a randomised controlled trial
}

\author{
Maria Waling* and Christel Larsson \\ Department of Food and Nutrition, Umeå University, SE-901 87 Umeä, Sweden
}

(Received 14 March 2012 - Final revision received 24 August 2012 - Accepted 24 August 2012)

Journal of Nutritional Science (2012), vol. 1, e16, page 1 of 11

doi:10.1017/jns.2012.17

Abstract

More knowledge about improving dietary intake in secondary preventive actions against childhood overweight and obesity is needed. The objective was to evaluate the impact of a 2-year intervention on energy, macronutrient and food intake of overweight and obese children participating in a randomised controlled trial. Children (8-12 years old) living in Sweden were recruited to participate for 2 years between 2006 and 2009 . The children were randomised into either an intervention group ( $n$ 58), participating in an intervention concerning food habits, physical activity and behavioural change, or a control group ( $n$ 47). Dietary intake at baseline and the 2-year measurement were assessed with a diet history interview covering $14 \mathrm{~d}$. Energy intake (EI) of the intervention and control groups was underestimated by 28 and $21 \%$, respectively, after 2 years, but with no difference between the groups $(P=0 \cdot 51)$. After 2 years of intervention, the intervention group, compared with the control group, had a lower intake of sugar-sweetened beverages $(P=0 \cdot 015)$ as well as a higher intake of foods high in fibre, low in saturated fat, sugar and salt $(P=0.031)$. Further, a lower EI in relation to BMR, lower total fat, MUFA and cholesterol was seen in the intervention group compared with the control group. In conclusion, the food and nutrient intake of overweight and obese children was improved after participating in a 2-year intervention programme. Dietary counselling should be included in secondary preventive actions against childhood overweight and obesity to promote healthy food habits.

Key words: Dietary intake: Overweight: Obesity: Randomised controlled trials

Over the last few years, the increased prevalence of overweight and obesity among children has stagnated and even decreased in some European countries ${ }^{(1,2)}$, including Sweden ${ }^{(3-5)}$. However, compared with 20 years ago there is still a high prevalence of overweight and obesity among children ${ }^{(6-8)}$. Genetic predisposition is an important contributory factor, but one that cannot explain the increased prevalence of overweight and obesity during the last few decades. An imbalance in energy intake (EI) and total energy expenditure (TEE) is considered to be the most important explanation for the development of overweight and obesity ${ }^{(9)}$. Decreased physical activity $^{(10)}$ in combination with increased portion sizes ${ }^{(11)}$, sweet-beverage consumption $^{(12)}$, as well as a higher intake of foods high in energy but low in nutrients (energy-dense foods $)^{(13)}$ are a few examples of how lifestyle has changed during the last few decades. The most recent Swedish national dietary assessment survey concluded that Swedish children of 8 and 11 years of age obtain $25 \%$ of their daily EI from energy-dense foods such as sweet beverages, sweets, chips, ice cream, desserts, cakes and biscuits ${ }^{(14)}$. These children consumed sweets three to five times per week and $13 \%$ of them ate sweets daily. Fruit and vegetable intake was on average 239 and $193 \mathrm{~g} / \mathrm{d}$, which is about half of the recommended amount for children $(>400 \mathrm{~g} / \mathrm{d}$ in 4-10-year-olds and $>500 \mathrm{~g} / \mathrm{d}$ in $\geq 10$-year-olds) ${ }^{(15)}$. Further conclusions were that Swedish children consume too little dietary fibre and too

Abbreviations: DHI, diet history interview; E\%, energy percentage of total intake; EI, energy intake; TEE, total energy expenditure.

* Corresponding author: Maria Waling, fax +46907869980, email maria.waling@kost.umu.se

(C) The Author(s) 2012. The online version of this article is published within an Open Access environment subject to the conditions of the Creative Commons Attribution-NonCommercial-ShareAlike licence <http://creativecommons.org/licenses/by-nc-sa/2.5/>. The written permission of 
much SFA, sucrose and salt compared with the national recommendations ${ }^{(15)}$. Perhaps due to difficulties in measuring dietary intake, there is little evidence that the food habits of overweight and obese children differ from those of their normal-weight peers.

So far, a few intervention studies on overweight and obese children have focused on evaluating the effect of both foodand nutrition-based recommendations given in the intervention $^{(16-19)}$. It is essential to study both food and nutrient intakes in order to gain a better understanding of how well food-based and nutritional recommendations may be adapted, and to ascertain whether consumption of different food groups can be changed. Information about food intake is valuable for both those changing their dietary habits and the nutritionists counselling them on how to improve their dietary intake. The objective of the present study was to evaluate the impact on energy, macronutrient and food intake of a 2-year food and physical activity intervention, aimed at improving lifestyle and health among overweight and obese children.

\section{Experimental methods}

\section{Participants}

In this randomised controlled trial children were invited through letters sent to families ( $n$ 6290) living in or near the northern Swedish university town of Umeå (about 114000 inhabitants). The recruitment procedure has been described in previous publications ${ }^{(20,21)}$, and inclusion criteria comprised: children born between 1995 and 1998, an age- and sexadjusted $\mathrm{BMI} \geq 25 \mathrm{~kg} / \mathrm{m}^{2(22)}$, and access to the Internet. Exclusion criteria were: whether a child had a chronic disease that affected metabolic variables (e.g. blood lipids, glucose and insulin) or had an attention deficit disorder diagnosis. Power calculations were done on the primary outcome variable (EI), and in order to be able to detect a $1000 \mathrm{~kJ} / \mathrm{d}$ difference between the intervention and control groups (11 100 v. 10100 (SD 1600) kJ, $\alpha=0.05$, power $80 \%$ ), a sample of forty-one participants per group was required.

The children participating in the present study were recruited at four different points in time, i.e. October 2006 and January, March and May 2007, and were randomly allocated to either an intervention or a control group. In total, 112 children proved to be interested in participating in the present study, and, of these, 105 (fifty-three girls and fifty-two boys) overweight and obese children met the inclusion criteria (Fig. 1). In total, ninety-two children participated in diet history interviews (DHI) at baseline, and after 2 years of participation, forty-eight of these participated in the DHI at endpoint measurements. The present study was conducted according to the guidelines laid down in the Declaration of Helsinki and all procedures involving human subjects were approved by the Regional Research Ethics Review Board, the Faculty of Medicine at Umeå University, Umeå, Sweden. Written informed consent was obtained from the children's parents. All children were individually coded and data were analysed anonymously.

\section{Intervention}

The intervention was designed as a family-based lifestyle programme in Sweden from 2006 to 2009, based on the principles of behavioural $^{(23,24)}$ and solution-oriented group work ${ }^{(25)}$. The first year of the intervention has been described in more detail in previous publications ${ }^{(20,21)}$. In summary, forty-eight intervention children were divided into four groups and invited to fourteen meetings one to two times per month during the first year. Each session lasted for 1-1.5 h and was held at the Department of Food and Nutrition at Umeå University. Five sessions had a theme concerning improvement of dietary intake, while the remaining sessions dealt with physical activity and behavioural change. The mean participation rate was seven sessions (range: 1-14) for the twenty-eight intervention children participating during the first year of the intervention. Of the twenty-eight children, four $(14 \%)$ attended all fourteen sessions of the intervention. In total, six out of the twenty-eight children $(21 \%)$ participated in all five sessions regarding food habits, 21 $\%$ in four, $29 \%$ in three, $21 \%$ in two and $<1 \%$ in one of the food sessions. Regarding the five sessions relating to behavioural change, eight out of the twenty-eight children (29\%) participated in all five sessions, $25 \%$ in four, $36 \%$ in three, $<1 \%$ in two and $<1 \%$ in one. The physical activity section of the intervention has been evaluated elsewhere ${ }^{(20)}$. Food-related advice given was based on national recommendations and guidelines when communicating with the children and parents on how to improve the child's food habits ${ }^{(15,26)}$. The main message to the children and parents with regard to food intake was: to eat more fruit, berries, vegetables and root vegetables, limit the intake of energy-dense foods $(\leq 10 \%$ energy of total intake; $\left.\mathrm{E}^{\%}\right)^{(26)}$ and choose keyhole-labelled foods ${ }^{(27)}$. The keyhole symbol has been devised by the Swedish Food Administration and is currently in use in Sweden, Norway and Denmark, as a guide for consumers to select food items that contain more dietary fibre and less salt, sugar and SFA compared with similar food items. It can be found on foods such as bread, dairy products and cold cuts. To increase fruit and vegetable intake, children and parents were introduced to the SAPERE-method, which aims to help children to increase preferences for new foods by using their senses to describe different foods ${ }^{(28)}$. They were also introduced to the 'plate model' which shows how a healthy meal can be composed of visualising proportions of different food groups (carbohydrate- and protein-rich foods, as well as vegetables, root vegetables and fruits) ${ }^{(29)}$. Furthermore, in order to decrease the intake of sugar-rich foods, one session was an exhibition where healthier alternatives to commonly consumed high-sugar foods were presented. Prior to each session, the children and sometimes the parents completed an assignment that related to the topic of the coming session. The assignments were of a kind where the children and parents themselves set individual goals based on the child's current situation and then made a plan as to how the goal was to be reached. They were also instructed to identify obstacles that could prevent the child from reaching the goal and how these could be prevented.

The second year of the intervention was Internet-based, with a web platform for communication between the leaders 


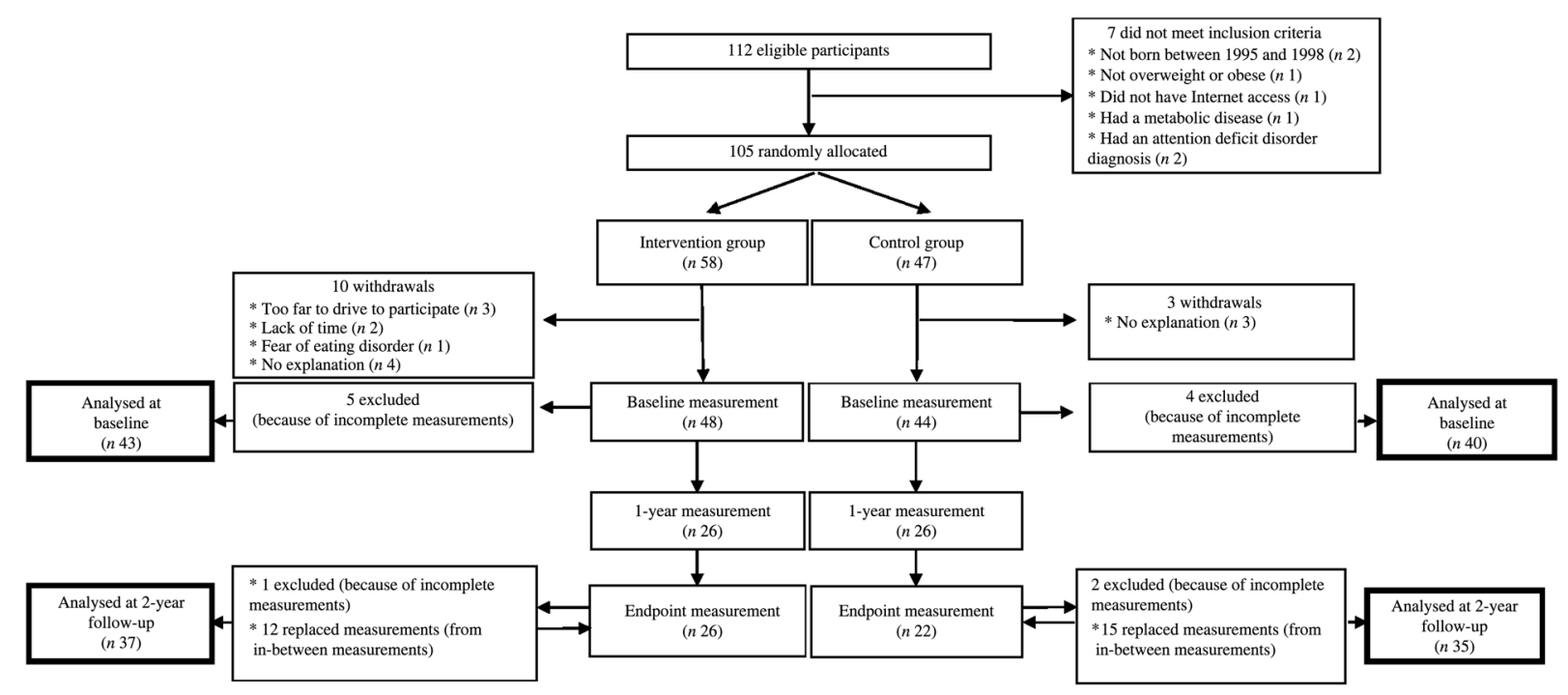

Fig. 1. Flowchart of the recruitment and participation of overweight and obese 10-year-old children in a 2-year randomised controlled trial.

and the participants, as well as among the latter. This section of the intervention was considered as a stage where the families worked on maintaining lifestyle changes that had been initiated during the first year of the intervention, as well as on making new ones. The second year was based on twelve assignments that the children, and sometimes the parents, were supposed to do at home and then submit to the leaders and/or other participants through the web platform. Of the assignments, five concerned food habits, while the others were related to physical activity and behavioural change. A total of twenty-six children participated throughout the second year of the intervention study and the mean participating rate of the assignments concerning food habits was eight for assignments 2 and 4, six for assignment 5 and three for assignments 7 and 10 .

In addition to their participation in the same measurements as the intervention group, the control group participated in only one initial information meeting about the measurements.

\section{Dietary assessment methods}

Diet history method. The DHI covering two previous weeks were conducted at baseline and after 2 years, in order to capture the participating children's habitual food intake. The DHI was performed during the same month of the year at baseline and at endpoint for each child. The DHI were conducted between October and June in 2006-2007 and 2008-2009. Each interview was conducted on a single occasion with the child accompanied by at least one of the parents. The portion sizes of each food item and dish eaten by the child were described with the help of food-portion photographs ${ }^{(30)}$, household measures, standard weights of food items, or bags of rice of different volumes. DHI conducted at baseline were performed by the first author $(n$ 73) and by a nutritionist trained by the first author ( $n$ 19). After 2 years, twelve DHI were conducted by the first author and the remaining thirty-six were completed by the same nutritionist and two dietitians trained by the first author. All trained interviewers followed a standardised interview protocol and observed two interviews performed by the first author before conducting interviews themselves. The DHI method used and its validity have been described in greater detail in a previous publication and the results showed that the DHI captured $86 \%$ of the TEE of the overweight and obese children at baseline ${ }^{(31)}$.

Food record method. To follow the children's food habits during the 2 years of their participation, each child was instructed to keep six separate 2-d food records during the 2 years of the study. In addition, after 1 year of participation, each child was instructed to keep a 4-d food record. The 2-d food records were scheduled to cover weekdays, weekends and the different seasons. The 4-d food records included consecutive days and covered two weekdays and two weekend days. To keep the food record, the children were provided with a digital camera and were instructed to photograph everything eaten during the days covered by the record. At the end of every such day, the child and one parent were asked to look at the photographs taken during that day and document everything consumed in a food diary. Portion sizes were assessed by the child and parent by viewing the photographs and comparing them with pictures in a food-portion picture book containing known portion weights ${ }^{(30)}$, household measures and standard weights of food items. The food record method used has been described in more detail in a previous publication ${ }^{(31)}$.

Intake of food, energy and macronutrients. Reported food intakes from the DHI at baseline and the 2-year measurement as well as the 2- and 4-d food records were entered in the dietary analysis program Dietist XP version 3.0 (Kost och Näringsdata $\mathrm{AB}$ ), in order to calculate the daily intake of energy and nutrients. Dietist XP uses the Swedish food composition database (version 6 March 
2008), which contains approximately 2000 different food items and dishes. When such an item or dish was not found in the database, a similar food item or dish in the database was entered. If no suitable food item or dish could be identified, information on the energy and nutrient content of that specific food or dish was manually entered in the database.

The nutritional calculation program used (Dietist XP) did not have a feature for placing individual foods in food groups, and therefore a program that could do this had to be developed in Excel (Microsoft, 2010). The program was designed so that the food groups, as well as the individual foods included in each group, could be selected by the user. The output from the program was the contribution of each food group in g, energy and nutrients to each individual's intake. The food groups devised in the present study were based on the 107 food groups that the database (version 2008-03-06), created by the Swedish Food Administration, comprised at that time. These 107 groups were reduced to fifty-one by merging groups, while a few new groups were also created, e.g. one for keyhole-labelled foods.

To evaluate the intervention effect on the intake of energy, macronutrients and food, the children's dietary intake was compared with objectively measured TEE, the national nutritional recommendations and guidelines on eating at least 500 $\mathrm{g} / \mathrm{d}$ of fruit, berries, vegetables and root vegetables (excluding potatoes), $\leq 10 \mathrm{E} \%$ of energy-dense foods, $\leq 10 \mathrm{E} \% \mathrm{SFA}, 2-3$ $\mathrm{g} / 1000 \mathrm{~kJ}$ dietary fibre and $\leq 10 \mathrm{E} \%$ refined sugar, e.g. sucrose, glucose, fructose and starch hydrolysates ${ }^{(15,26)}$. Sucrose was the only refined sugar that could be calculated using the Swedish food composition database and in the analyses it therefore represents refined sugar.

Measurement of total energy expenditure and anthropometrics. To assess the children's TEE, a SenseWear armband Pro 2 and 3 (BodyMedia Inc.) was used during the corresponding days when dietary intake was assessed. Even though two different hardware models were used (SenseWear armband Pro 2 and 3), the same software version was used (Innerview professional software version 5.1) and the same variables were analysed for the two models. At baseline and endpoint measurements, TEE was measured during a $4-\mathrm{d}$ period included in the $14-\mathrm{d}$ period that the DHI covered. TEE was also measured during the same days as six separate food records were kept during the study. The armband has been previously described and validated, and has shown good validity with regard to measuring TEE in groups of overweight and obese children $^{(32)}$. In the analysis of energy, macronutrient and food intake, children with an EI:TEE quotient of less than 0.6 were excluded, since it was regarded by the authors as an incomplete measurement.

The children's weight and height were measured by two paediatric nurses at baseline, after 1 year and at endpoint. BMR was calculated according to Schofield ${ }^{(15)}$ for normalweight children and Dietz et al. ${ }^{(33)}$ for overweight and obese children.

\section{Statistical analysis}

Statistical analyses were conducted using SPSS for Windows version 18.0 (SPSS Inc.). To evaluate energy and macronutrient intake, the following parametric tests were used (all variables were normally distributed): a paired-sample $t$ test for the evaluation of changes within groups from baseline to endpoint, and an independent $t$ test for comparison of differences in changes from baseline to endpoint between the groups, as well as for the evaluation of differences between groups at baseline. For the evaluation of the effect at endpoint between the groups, a one-way ANCOVA, using the baseline measurements as the covariates, was conducted. For the evaluation of all food groups, which were not normally distributed, the following non-parametric tests were used: the Wilcoxon signed rank test for the evaluation of changes within groups from baseline to endpoint, and the Mann-Whitney $U$ test for comparison of differences in changes from baseline to endpoint between the groups as well as for the evaluation of differences between groups at baseline and endpoint. The results were considered statistically significant if the two-tailed $P$ value was $<0 \cdot 05$.

Results were analysed on an intention-to-treat analysis basis, which means that all children were analysed in the group which they were originally randomised. The principle of carrying the last observation forward was followed for children who dropped out before endpoint measurements, as well as for those who had an EI:TEE quotient $<0.6$ at the endpoint measurement. For missing or incomplete measures at endpoint, observations that had an EI:TEE quotient $\geq 0.6$ from the latest food records were carried forward and used as endpoint measures. All analyses were also performed as per protocol to see whether the results differed. Since there were no differences between the two approaches, we chose to present the results where the last observation carried forward principle was followed.

\section{Results}

\section{Participants}

Of the ninety-two children who participated in the DHI at baseline, eighty-three children had complete measurements (an EI:TEE quotient $>0 \cdot 6$ ) and were included in the analysis (Fig. 1). Of the forty-eight children who participated in the DHI at endpoint, forty-five children had complete measures. Dietary intake data for the three children with incomplete measurements were replaced by data from the last complete food records 2, 18 and 20 months, respectively, before the endpoint measurements. The same principle of carrying the last observation forward was followed for twenty-four of the forty-four withdrawals between baseline and endpoint, where 2 - and 4-d food records were used from an average of 16 months (range: 2-22 months) before the endpoint measurement. This left seventy-two children to be included in the final analysis at endpoint. The forty-five children with complete DHI data at the 2-year measurement did not differ in baseline background characteristics or baseline dietary intake compared with baseline data of those thirty-eight 
children who dropped out before endpoint measurements or were excluded because of incomplete DHI at endpoint (data not shown).

At endpoint there were thirty-eight children who participated in both the DHI and the TEE measurements by a SenseWear armband during the same time period, and these were included in the comparison of estimated EI with objectively measured TEE.

\section{Evaluation of estimated energy intake}

The correlation coefficient between EI and TEE at endpoint was $0.30(P=0.07)$ and the mean difference between EI and TEE is illustrated in a Bland-Altman plot (Fig. 2). The EI was $25 \%$ lower than the measured TEE of the group as a whole $(P<0 \cdot 001)$. In the intervention and control groups, EI was $28 \%(P<0.001)$ and $21 \%(P<0.001)$ lower, respectively, than measured TEE (Table 1), with no differences between intervention and control groups $(P=0.111)$ or between sexes $(P=0.446)$ with regard to the comparison of EI and TEE. Compared with TEE, the EI was $41 \%(P=$ $0.004), 24 \%(P<0.001)$ and $15 \%(P=0.007)$ lower among the obese, overweight and normal-weight children, respectively. Furthermore, a strong negative correlation was found between the BMI and the degree of difference between EI and TEE $(r-0.50, P \leq 0 \cdot 001)$. This indicates that the children with a higher BMI at endpoint had a lower EI than TEE, compared with children with a lower BMI. There was no significant correlation between age and difference between EI and TEE $(r 0 \cdot 28, P=0.09)$ at endpoint.

\section{Energy and macronutrient intake}

At baseline there were no differences in background characteristics, energy or macronutrient intake between intervention and control groups or between the sexes ${ }^{(21)}$. However, at endpoint, the intervention group had a lower EI:BMR quotient compared with the control group $(P=0.040)$ (Table 2). Furthermore, from baseline to endpoint measurement the intervention group decreased their daily EI $(P=0.002)$ and the quotient EI:TEE $(P=0.003)$, while no statistical significant change were seen in the control group.

At endpoint the intervention group ate $16 \%$ less fat $(\mathrm{g})$ $(P=0.022), 16 \%$ less MUFA $(\mathrm{g})(P=0.048)$ and $22 \%$ less cholesterol (mg) $(P=0.035)$ than the control group (Table 2). Furthermore, from baseline to endpoint measurement the intervention group had a statistically significant decrease in their intake of fat $(\mathrm{g})$, SFA $(\mathrm{g})$, cholesterol $(\mathrm{mg})$ and protein $(\mathrm{g})$, while the intake of the control group remained unchanged. The control group, on the other hand, decreased their carbohydrate intake $(\mathrm{E} \%)$ but increased their fat $(\mathrm{E} \%)$, MUFA (E\%) and PUFA (E\%) intake statistically significant from baseline to endpoint. The intake of the intervention group remained unchanged in this regard. In both groups carbohydrates (g), monosaccharides (g) and disaccharides (g) showed a statistically significant decrease from baseline to endpoint.

\section{Food intake}

At baseline the intervention group had a $74 \%$ higher intake of sugar-sweetened beverages (soft drinks and unsweetened fruit juices) compared with the control group $(P=0.014)$ (Table 3). However, at endpoint the opposite situation was seen, and the intervention children had an $89 \%$ lower intake of sugar-sweetened beverages than the control group ( $P=$ $0 \cdot 015)$. In addition to the lower intake of sugar-sweetened beverages, the intake of energy-dense foods was $53 \%$ lower in the intervention than the control groups at endpoint $(P=0 \cdot 011)$. Furthermore, the intervention group ate more keyhole-labelled foods $(P=0.031)$, especially dairy products $(3.5$ times more $)$ $(P=0.032)$ than the control group. There was no difference in the consumption of breads ( $>6 \%$ fibre) between the intervention and control groups. However, when bread intake was grouped into crisp breads and soft breads, the intervention group consumed more of the former as compared with the control group at the 2-year measurement $(P=0 \cdot 028)$.

There was a difference in change from baseline to endpoint between the two groups in respect of the consumption of both energy-dense foods $(P=0.008)$ and sugar-sweetened beverages $(P=0.003)$, which may be explained by an increased consumption in the control group (Table 3). The control group's intake of energy-dense foods increased by $60 \%(P=0.043)$ from baseline to endpoint and sweet beverages increased by $92 \%(P=0.009)$ during the same period, while no significant change was seen in the intervention group. The intervention group decreased their intake of sweets from baseline to endpoint measurement by $46 \%(P=0 \cdot 048)$, while no significant change was seen in the control group (Table 3). From baseline to endpoint measurement, the control group decreased their intake of sugar-free sweets $(P=0.035)$ and keyhole-labelled foods $(P \leq 0 \cdot 001)$, while no significant change was seen in the intake of the intervention group during the 2 -year study period.

\section{Discussion}

After 2 years of participation, the children in the intervention group had a lower intake of sugar-sweetened beverages as well as a higher intake of keyhole-labelled foods, primarily dairy products, compared with the control group. Furthermore, a lower intake of EI in relation to BMR, total fat, MUFA and cholesterol was seen in the intervention group compared with the control group.

\section{Evaluation of estimated energy and macronutrient intake}

At baseline the EI of the children was underestimated by $14 \%$ and there was no difference between intervention and control groups $^{(31)}$. Neither were there any differences between the groups at endpoint, but at this time the EI was 28 and $21 \%$ lower than the TEE of the intervention and control groups, respectively. It is not possible to say how much of the difference between groups at endpoint is caused by undereating due to participation in an intervention study aimed at decreasing EI and increasing TEE and/or an increased underestimation of EI, but most likely it is a combination of both. 


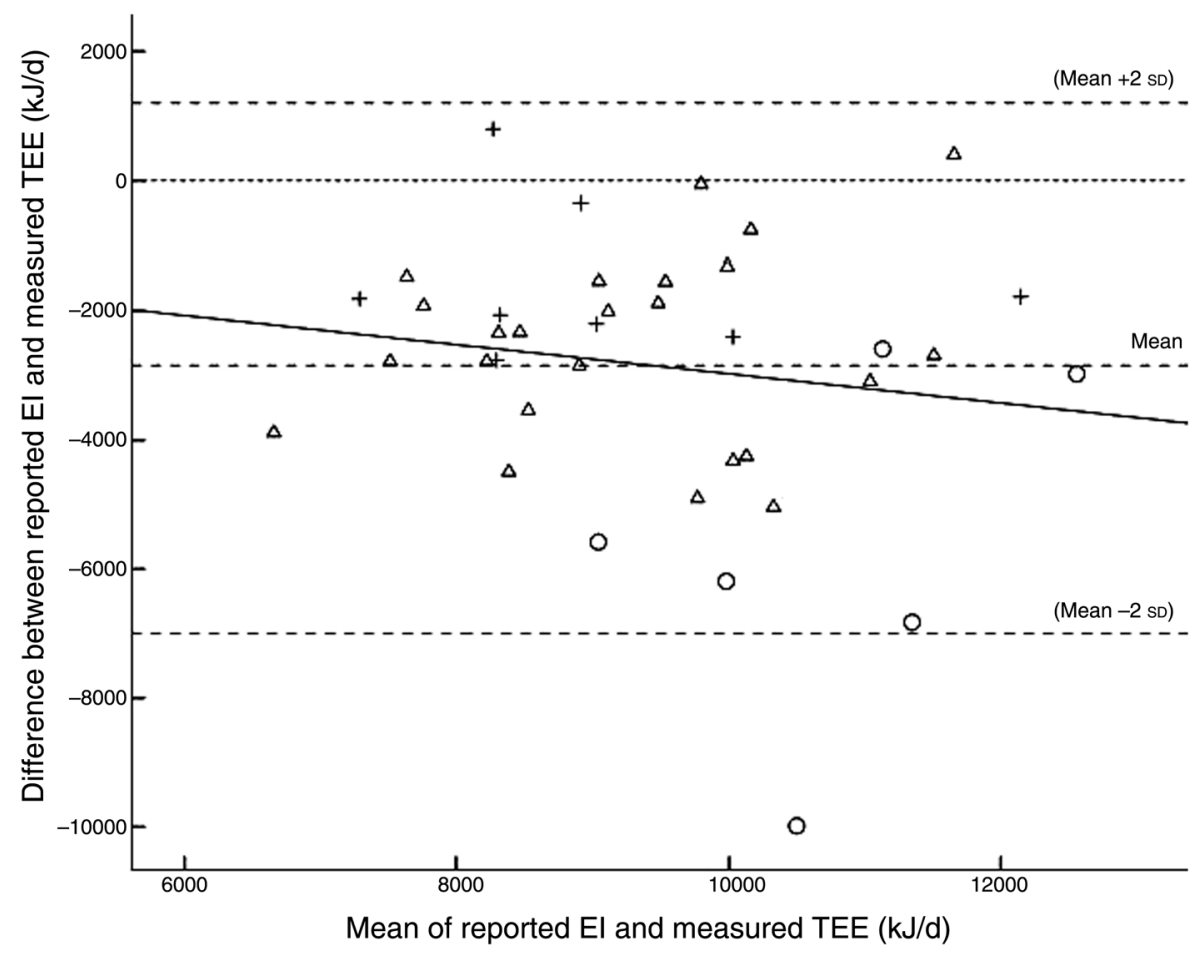

Fig. 2. Differences between reported energy intake (EI) in diet history interviews at endpoint, of normal-weight (+), overweight $(\Delta)$ and obese (o) children $(n 38)$ and their total energy expenditure (TEE) measured by a SenseWear armband, against the mean of the two variables. The correlation coefficient was $0.153(P=0.360)$ and the linear regression equation was $y=-0.71-0.23 x$.

SFA intake (13.4 and $14.1 \mathrm{E} \%$ in intervention and control groups, respectively) was above the maximum recommended intake $(\leq 10 \mathrm{E} \%)^{(15)}$, which is similar to the intake presented in other studies including normal-weight, overweight and obese children ${ }^{(34,35)}$. The proportional intake of carbohydrates $(51.7$ and $50.6 \mathrm{E} \%$ in intervention and control groups, respectively) was almost the same in the present study as the intake found in other studies among normal-weight children (52 and $53 \mathrm{E} \%$ among 9- and 11-year-old children) ${ }^{(14,36)}$ and similar to the $51 \mathrm{E} \%$ intake found in 9-year-old overweight children ${ }^{(35)}$. Sucrose intake was close to the maximum recommended intake $(\leq 10 \mathrm{E} \%)$ in both groups, but is lower than that shown in the latest dietary survey of a national sample of 8- and 11-year-old Swedish children (12 E\%). The children in the present study had a somewhat higher intake of dietary fibre $(2.03 \mathrm{~g} / 1000 \mathrm{~kJ}$ in the intervention group and $1.85 \mathrm{~g} / 1000 \mathrm{~kJ}$ in the control group) than reported in another study, $1.6 \mathrm{~g} / 1000 \mathrm{~kJ}$ among overweight children and $1.7 \mathrm{~g} / 1000 \mathrm{~kJ}$ among normal-weight children ${ }^{(35)}$. So far there are a limited number of studies that have evaluated different types of macronutrient composition in the treatment of overweight and obesity among children and no consensus can be reached as to their effectiveness ${ }^{(37,38)}$.

\section{Food intake}

The EI from energy-dense foods was above recommendations $(\leq 10 \mathrm{E} \%)$ both at baseline and at endpoint in both groups. At

Table 1. Reported energy intake (EI) in diet history interviews (DHI) and measured total energy expenditure (TEE) by a SenseWear armband (SW) at endpoint in overweight and obese children participating in a 2-year randomised controlled trial* (Mean values and standard deviations)

\begin{tabular}{|c|c|c|c|c|c|c|c|c|c|c|}
\hline & \multicolumn{2}{|c|}{ Girls ( $n$ 19) } & \multicolumn{2}{|c|}{ Boys ( $n 19)$} & \multirow[b]{2}{*}{$P$} & \multicolumn{2}{|c|}{$\begin{array}{l}\text { Intervention group } \dagger \\
(n 21)\end{array}$} & \multicolumn{2}{|c|}{$\begin{array}{l}\text { Control group } \ddagger \\
(n 17)\end{array}$} & \multirow[b]{2}{*}{$P$} \\
\hline & Mean & SD & Mean & SD & & Mean & SD & Mean & SD & \\
\hline BMRß (kJ/d) & 7180 & 800 & 7430 & 1070 & 0.461 & 7590 & 1000 & 6950 & 960 & 0.052 \\
\hline $\mathrm{El}_{\mathrm{DHI}}(\mathrm{kJ} / \mathrm{d})$ & 7700 & 1070 & 8340 & 19990 & 0.225 & 7860 & 1280 & 8210 & 1970 & 0.515 \\
\hline $\mathrm{TEE}_{\mathrm{sw}}(\mathrm{kJ} / \mathrm{d})$ & 10300 & 1340 & 11400 & 2160 & 0.055 & 1120 & 1860 & 1050 & 1860 & 0.233 \\
\hline $\mathrm{El}_{\mathrm{DH} / \mathrm{BMR}}$ & 1.09 & 0.22 & 1.13 & 0.30 & 0.571 & 1.05 & 0.18 & 1.20 & 0.32 & 0.515 \\
\hline $\mathrm{TEE}_{\mathrm{SW}} / \mathrm{BMR}$ & 1.45 & 0.22 & 1.55 & 0.24 & 0.185 & 1.48 & 0.23 & 1.51 & 0.25 & 0.698 \\
\hline $\mathrm{El}_{\mathrm{DH}} / \mathrm{TEE}_{\mathrm{SW}}$ & 0.75 & 0.09 & 0.75 & 0.20 & 0.906 & 0.72 & 0.15 & 0.79 & 0.16 & 0.154 \\
\hline
\end{tabular}

* Differences between groups and sexes were tested with Student's $t$ test for independent samples.

+ Girls, $n$ 8; boys, $n 13$.

$\ddagger$ Girls, $n 11$; boys, $n 6$.

$\S$ BMR was calculated according to Schofield ${ }^{(15)}$ for normal-weight children and Dietz et al. ${ }^{(33)}$ for overweight and obese children. 


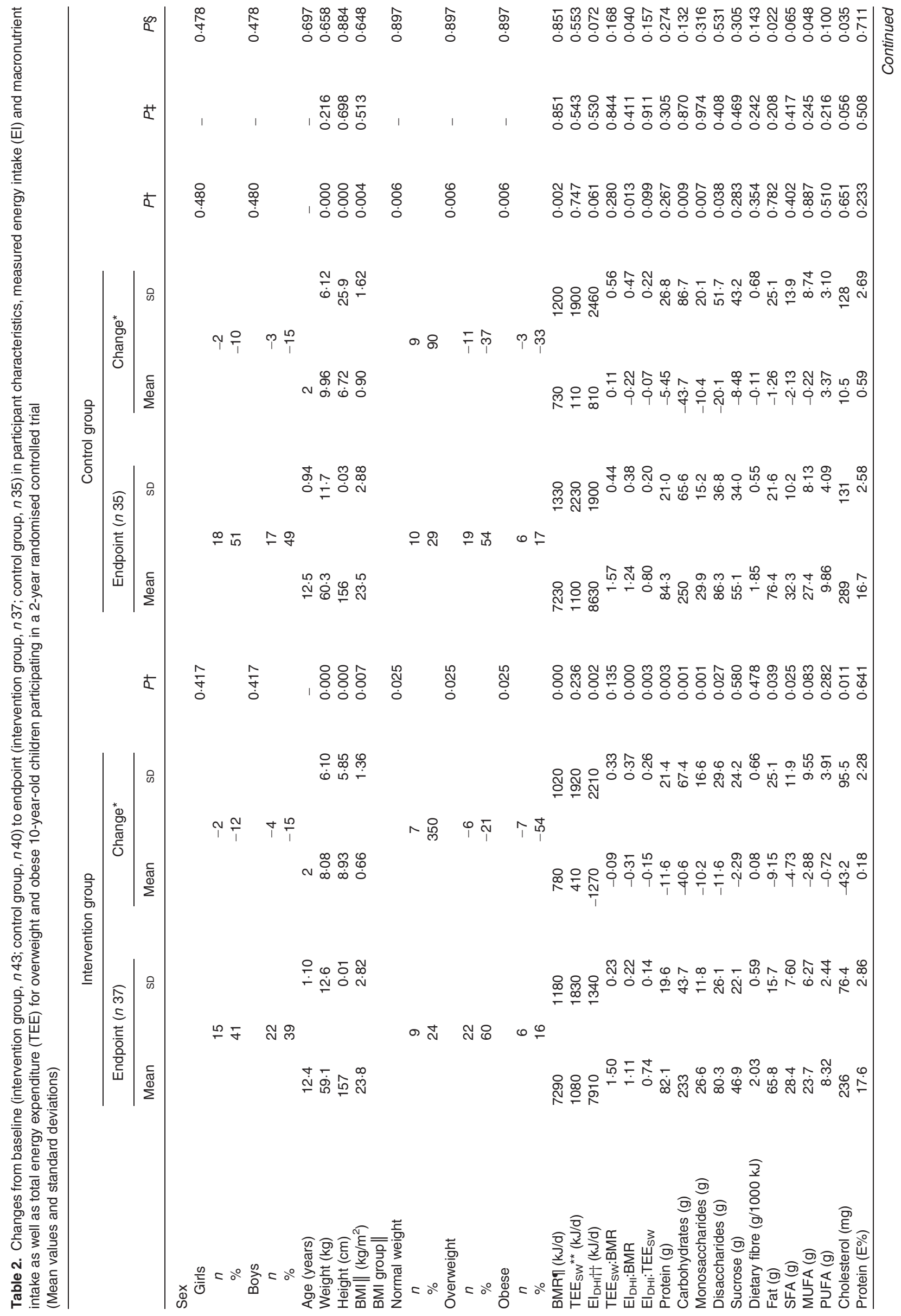


baseline the intervention group had a much higher intake of sugar-sweetened beverages than the control group. However, this converged during the 2-year intervention period and at endpoint the intervention group had a much lower intake than the control group. The converged results and the difference are explained by an increased consumption of sugar-sweetened beverages in the control group. During the 2 -year study period the intervention group showed a nonsignificant decrease in sugar-sweetened beverage consumption, which did not result in any lower proportion achieving the maximum recommended intake of energy-dense foods at endpoint compared with the control group. It is possible that the children in the intervention group would have to decrease their consumption of other energy-dense food groups as well in order to be able to show a decreased proportion of children reaching the maximum recommendation of $10 \mathrm{E} \%$ from energy-dense foods. The intake of energy-dense foods in the present study (14 and $17 \mathrm{E} \%$ in intervention and control groups, respectively, at endpoint) was lower than that found in a national sample of Swedish children, where the intake of energy-dense foods was as high as $25 \mathrm{E}^{\%}{ }^{(14)}$ but higher than that reported among obese 9-year-old children in Sweden $(9 \cdot 7 \mathrm{E} \%)^{(35)}$. The role of sugar-sweetened beverages in the development of overweight and obesity has frequently been discussed, but so far no true consensus has been reached $^{(39-42)}$. Since the cause of overweight and obesity is multifactorial, future prevention and treatment will most likely need to address more than one risk factor ${ }^{(40-42)}$.

No significant change was found in the fruit, berry, vegetable and root vegetable intake of either the intervention or the control groups during the 2-year study period. This result deviates from other studies, which have noted a tendency towards a decreased intake of fruit and vegetables with increasing age ${ }^{(14,43)}$. A possible reason for this could be that children in both groups were affected by participating in a study focusing on dietary intake. Even though their intake did not decrease, the fruit and vegetable intake was low compared with national guidelines both at baseline and endpoint. These intake levels are also similar to the fruit and vegetable intake shown among Swedish 11-year-old children who consumed $193 \mathrm{~g} / \mathrm{d}$ of fruit and vegetables ${ }^{(14)}$ and among 11 -year-olds in nine other European countries who consumed $145-265 \mathrm{~g} / \mathrm{d}^{(44)}$.

The intervention in the present study appears to have had a maintaining effect on the proportion of keyhole-labelled foods consumed among the children in the intervention group. The control group, on the other hand, decreased their intake from baseline to endpoint, which resulted in the intervention group having a higher intake of keyhole-labelled foods at endpoint measurement. To date, no research has been undertaken to evaluate the effect of keyhole-labelled foods in intervention studies aimed at improving food habits of overweight and obese children.

\section{Methodological considerations}

The advantages of the present study are the relatively long measurement period, the validation of the DHI and the fact 


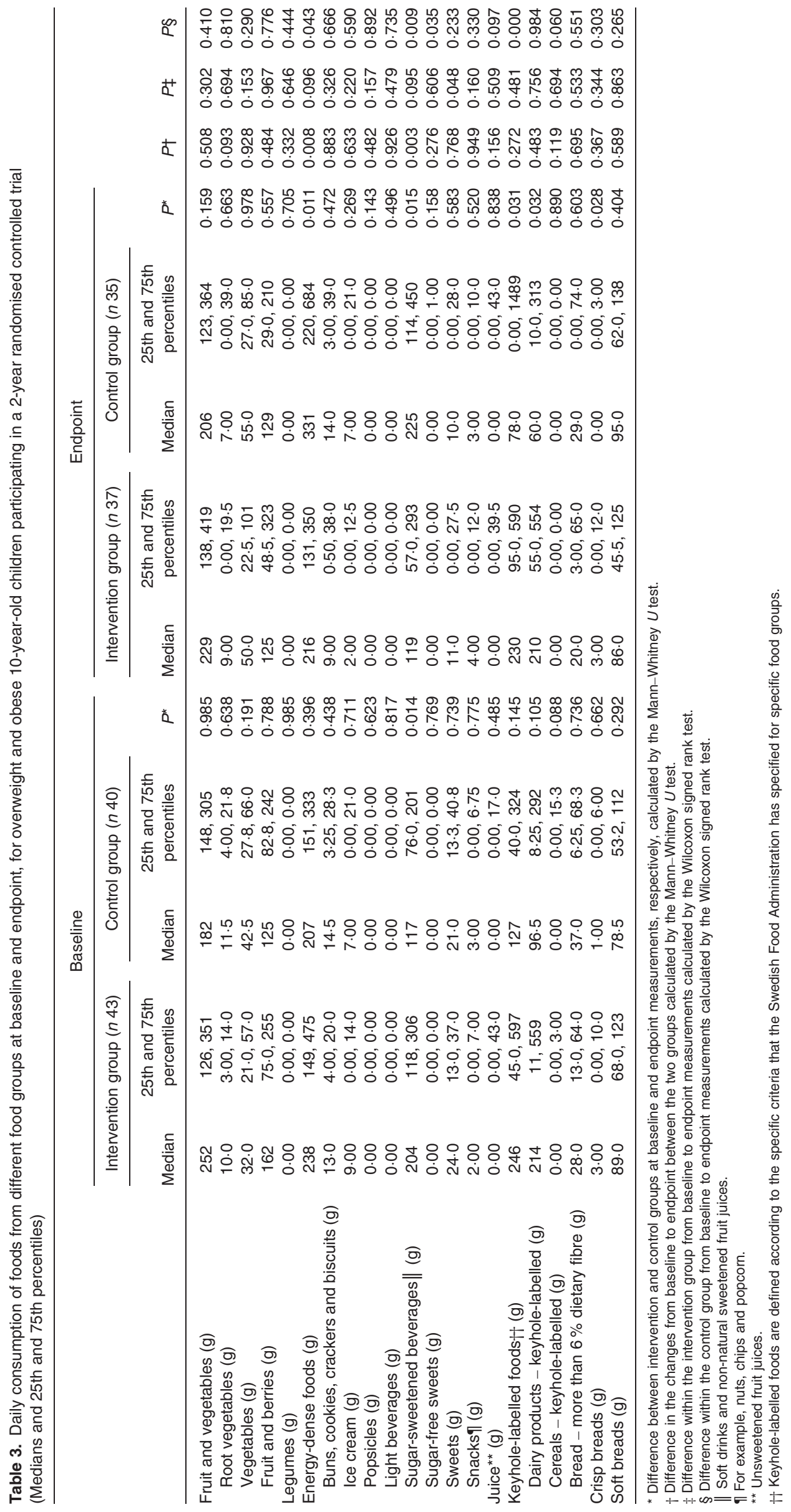


that a control group was included. One disadvantage was the $45 \%$ dropout rate during the 2 -year study period. This dropout rate is high but not uncommon in intervention studies aimed at treating childhood overweight and obesity according to a recent review where dropout rates varying between 12 and $52 \%$ were reported as early as at 1 -year follow-up ${ }^{(45)}$. A questionnaire was sent to all participants who dropped out, with the aim of obtaining more information about the dropouts as a group. Unfortunately, only fourteen of the total forty-two dropouts during the whole study returned the questionnaire, which made it difficult to perform any statistical analysis on the results. As a consequence of the high dropout rate and the consequently low study power, it is difficult to detect small differences between intervention and control groups. To increase the study power, a last observation carried forward strategy was used to replace dropouts, as well as incomplete measures. The last observation carried forward principle is one of the most commonly used strategies for dealing with dropouts $^{(46)}$ even though it has disadvantages such as diluting the effect of the intervention and concealing the within-subject variation $^{(47)}$.

Possible reasons as to why all food-related goals were not reached are attendance rate at sessions during year 1 of the intervention, the way of counselling and the web platform used during the second year of the intervention. No statistically significant differences were seen in results depending on attendance rate at the sessions (results not presented). However, the overall attendance rate of the sessions was relatively low, which probably diminished the effect of the intervention. The low attendance rates could be a result of too many sessions, sessions that were not appealing enough, or that the families put a low priority on participating. It is also possible that a stricter programme, e.g. more focus on advice on limiting EI and increasing TEE, or more individual counselling would have resulted in fulfilling more goals. The web platform used in the second year of the intervention was not used by the families as frequently as was planned in the study and consequently many families did not receive the intervention as it was planned the second year. The authors' understanding was that many parents were not comfortable in using a computer in general, and that many children were not familiar with using a computer in a communicative way.

The study population comprised families representing those living in the town of Umeå, Sweden, which is a town with a high educational level. A small proportion came from villages surrounding Umeå and only a small proportion of children with other ethnic backgrounds was represented. These are limiting factors when it comes to the generalisability of the study. However, major efforts were made to recruit as large and as generalisable a study population as possible, by contacting and inviting all eligible children living in or near Umeå ( $n$ 6290) to participate in the present study.

\section{Conclusions}

After 2 years of intervention the children in the intervention group, in contrast with the control group, had a lower intake of sweet beverages and a higher intake of keyhole-labelled foods, especially dairy products. Furthermore, the intervention group had a lower intake of total fat, MUFA and cholesterol compared with the control group. The present study shows that it is possible to improve the dietary habits of overweight and obese children after a 2-year lifestyle intervention. Dietary counselling for young children with overweight and obesity is important to promote healthy food habits and should be included in future strategies for the secondary prevention of overweight and obesity among children.

\section{Acknowledgements}

We would like to thank Emma Ådén, Anna Tellström and Tove Thegerström for their help in conducting interviews as well as with data entry. Furthermore, we would like to thank Professor Olle Hernell and Torbjörn Lind for their support in writing this paper, as well as Hans Stenlund and Marie Lindkvist for statistical advice. The authors' contribution to the work was as follows: M. W. contributed to the design of the study, conducted major parts of the intervention and data collection, analysed and interpreted the data and wrote major parts of the manuscript. C. L. designed the study, contributed to the intervention and data collection and took part in the interpretation of the data and writing the manuscript. Both authors read and approved the final version of the paper. M. W. had primary responsibility for the final content of the paper. M. W. and C. L. have no conflicts of interest. This study was supported by grants from the Vardal Foundation for Healthcare Sciences and Allergy Research; the Swedish Research Council for Environment, Agricultural Sciences and Spatial Planning; the Swedish Research Council; the Medical Faculty and the Faculty of Social Sciences at Umeå University; Västerbotten County Council; Dr PersFood AB; Majblommans Riksförbund; and the Magnus Bergvall Foundation.

\section{References}

1. Aeberli IAR, Knabenhans M \& Zimmermann MB (2008) The national prevalence of overweight in school-age children in Switzerland has decreased between 2002 and 2007. Int J Obes 32, Suppl. 1, S214.

2. Salanave B, Peneau S, Rolland-Cachera MF, et al. (2009) Stabilization of overweight prevalence in French children between 2000 and 2007. Int J Pediatr Obes 4, 66-72.

3. Sjöberg A, Lissner L, Albertsson-Wikland K, et al. (2008) Recent anthropometric trends among Swedish school children: evidence for decreasing prevalence of overweight in girls. Acta Paediatr 97, 118-123.

4. Sundblom E, Petzold M, Rasmussen F, et al. (2008) Childhood overweight and obesity prevalences levelling off in Stockholm but socioeconomic differences persist. Int J Obes (Lond) 32, 1525-1530.

5. Lager A, Fossum B, Rörvall G, et al. (2009) Children's overweight and obesity: local and national monitoring using electronic health records. Scand J Public Health 37, 201-205.

6. Petersen S, Brulin C \& Bergström E (2003) Increasing prevalence of overweight in young schoolchildren in Umeå, Sweden, from 1986 to 2001. Acta Paediatr 92, 848-853.

7. Ekblom O, Oddsson K \& Ekblom B (2004) Prevalence and regional differences in overweight in 2001 and trends in BMI distribution in Swedish children from 1987 to 2001. Scand J Public Health 32, 257-263. 
8. Mårild S, Bondestam M, Bergström R, et al. (2004) Prevalence trends of obesity and overweight among 10-year-old children in western Sweden and relationship with parental body mass index. Acta Paediatr 93, 1588-1595.

9. Goran MI \& Treuth MS (2001) Energy expenditure, physical activity, and obesity in children. Pediatr Clin North Am 48, 931-953.

10. Jimenez-Pavon D, Kelly J \& Reilly JJ (2010) Associations between objectively measured habitual physical activity and adiposity in children and adolescents: systematic review. Int J Pediatr Obes 5, $3-18$.

11. Ledikwe JH, Ello-Martin JA \& Rolls BJ (2005) Portion sizes and the obesity epidemic. J Nutr 135, 905-909.

12. Nielsen SJ \& Popkin BM (2004) Changes in beverage intake between 1977 and 2001. Am J Prev Med 27, 205-210.

13. Nielsen SJ, Siega-Riz AM \& Popkin BM (2002) Trends in energy intake in U.S. between 1977 and 1996: similar shifts seen across age groups. Obes Res 10, 370-378.

14. The Swedish National Food Administration (2006) Riksmaten-barn 2003 Livsmedels- och näringsintag bland barn $i$ Sverige (Food and Nutrient Intake Among Swedish Children). Uppsala: Livsmedelsverket.

15. Nordiska ministerrådet (2004) Nordic Nutrition Recommendations 2004: Integrating Nutrition and Physical Activity, 4th ed. Copenhagen: Nordic Council of Ministers.

16. Agras WS \& Mascola AJ (2005) Risk factors for childhood overweight. Curr Opin Pediatr 17, 648-652.

17. Moreno LA \& Rodriguez G (2007) Dietary risk factors for development of childhood obesity. Curr Opin Clin Nutr Metab Care 10, 336-341.

18. Newby PK (2007) Are dietary intakes and eating behaviors related to childhood obesity? A comprehensive review of the evidence. $J$ Law Med Ethics 35, 35-60.

19. Han JC, Lawlor DA \& Kimm SY (2010) Childhood obesity. Lancet $375,1737-1748$.

20. Bäcklund C (2010) Promoting physical activity among overweight and obese children. Effects of a family-based lifestyle intervention on physical activity and metabolic markers. Doctoral Thesis. Umeå University.

21. Waling M, Lind T, Hernell O, et al. (2010) A one-year intervention has modest effects on energy and macronutrient intakes of overweight and obese Swedish children. J Nutr 140, 1793-1798.

22. Cole TJ, Bellizzi MC, Flegal KM, et al. (2000) Establishing a standard definition for child overweight and obesity worldwide: international survey. $\mathrm{Br}$ Med J 320, 1240-1243.

23. Stunkard AJ \& Berthold HC (1985) What is behavior therapy? A very short description of behavioral weight control. Am J Clin Nutr 41, 821-823.

24. Robinson TN (1999) Behavioural treatment of childhood and adolescent obesity. Int J Obes Relat Metab Disord 23, Suppl. 2, S52-S57.

25. Sharry J (2007) Solution-Focused Group Work. Los Angeles: SAGE.

26. National Food Administration (2007) Bra mat $i$ skolan (Good Food in Schools). Uppsala: Elanders Berlings, Malmö.

27. Lindvall C (1992) Hur används nyckelhålsmärkningen av konsumenterna? (How is the key hole label used by consumers?). Var föda 44, 199-205.

28. Jonsson I, Gustafsson I-B \& Hallberg L (2000) Ett lustfyllt lärande om mat - Utvärdering av SAPERE-metoden (Pleasurable Learning about Food - Evaluation of the SAPERE Method). Örebro: Institutionen för Restaurang och Måltidskunskap Grythyttan.
29. Lindvall C (1992) Tallriksmodellen - ett bra instrument för kostrådgivning (The plate model - a good instrument for dietary counseling). Vår Föda 44, 238-241.

30. Håglin L, Hagman U \& Nilsson M (1995) Evaluation of the meal model "Matmallen": a means of estimating consumed amounts of food. Scand J Nutr 39, 79-83.

31. Waling MU \& Larsson CL (2009) Energy intake of Swedish overweight and obese children is underestimated using a diet history interview. J Nutr 139, 522-527.

32. Bäcklund C, Sundelin G \& Larsson C (2009) Validity of an armband measuring energy expenditure in overweight and obese children. Med Sci Sports Exerc 42, 1154-1161.

33. Dietz WH, Bandini LG \& Schoeller DA (1991) Estimates of metabolic rate in obese and nonobese adolescents. J Pediatr 118, 146-149.

34. Himes JH, Ring K, Gittelsohn J, et al. (2003) Impact of the pathways intervention on dietary intakes of American Indian schoolchildren. Prev Med 37, S55-S61.

35. Villa I, Yngve A, Poortvliet E, et al. (2007) Dietary intake among under-, normal- and overweight 9- and 15-year-old Estonian and Swedish schoolchildren. Public Health Nutr 10, 311-322.

36. Patterson E, Warnberg J, Kearney J, et al. (2009) The tracking of dietary intakes of children and adolescents in Sweden over six years: the European Youth Heart Study. Int J Behav Nutr Pbys Act 6, 91 .

37. Gibson LJ, Peto J, Warren JM, et al. (2006) Lack of evidence on diets for obesity for children: a systematic review. Int J Epidemiol 35, 1544-1552.

38. Sacks FM, Bray GA, Carey VJ, et al. (2009) Comparison of weight-loss diets with different compositions of fat, protein, and carbohydrates. N Engl J Med 360, 859-873.

39. Vartanian LR, Schwartz MB \& Brownell KD (2007) Effects of soft drink consumption on nutrition and health: a systematic review and meta-analysis. Am J Public Health 97, 667-675.

40. Forshee RA, Anderson PA \& Storey ML (2008) Sugar-sweetened beverages and body mass index in children and adolescents: a meta-analysis. Am J Clin Nutr 87, 1662-1671.

41. Gibson S (2008) Sugar-sweetened soft drinks and obesity: a systematic review of the evidence from observational studies and interventions. Nutr Res Rev 21, 134-147.

42. Libuda L \& Kersting M (2009) Soft drinks and body weight development in childhood: is there a relationship? Curr Opin Clin Nutr Metab Care 12, 596-600.

43. Talvia S, Rasanen L, Lagstrom H, et al. (2006) Longitudinal trends in consumption of vegetables and fruit in Finnish children in an atherosclerosis prevention study (STRIP). Eur J Clin Nutr 60, 172-180.

44. Yngve A, Wolf A, Poortvliet E, et al. (2005) Fruit and vegetable intake in a sample of 11-year-old children in 9 European countries: the Pro Children Cross-sectional Survey. Ann Nutr Metab 49, 236-245.

45. Oude Luttikhuis H, Baur L, Jansen H, et al. (2009) Interventions for treating obesity in children. Cochrane Database Syst Rev CD001872.

46. Hollis S \& Campbell F (1999) What is meant by intention to treat analysis? Survey of published randomised controlled trials. $\mathrm{Br} \mathrm{Med} J$ 319, 670-674.

47. Lachin JM (2000) Statistical considerations in the intent-to-treat principle. Control Clin Trials 21, 167-189. 